\title{
Asymptomatic Colonic Metastases of Lung Cancer: A Case Report
}

\author{
Pasquale Apolito ${ }^{1 *}$, Marco Bassi ${ }^{1}$, Stefania Ghersi ${ }^{1}$, Emanuele Dabizzi $^{1}$, Stefano Landi ${ }^{1}$, Adele Fornelli ${ }^{2}$ and \\ Vincenzo Cennamo ${ }^{1}$ \\ ${ }^{1}$ Gastroenterology and Interventional Endoscopy Unit, Local Health Authority of Bologna, Italy
}

${ }^{2}$ Pathology Unit, AUSL Bologna, Bellaria-Maggiore Hospital, Ital

Submission: March 30, 2021; Published: April 23, 2021

*Corresponding author: Pasquale Apolito, M.D. -Unit of Gastrointestinal and Interventional Endoscopy Unit, Surgical Department, AUSL Bologna, Maggiore Hospital, Largo Nigrisoli 2, 40139 Bologna, Italy

\section{Abstract}

Lung cancer is a malignant neoplasm that is frequently associated with distant metastasis. However, gastrointestinal tract is a rare location for lung cancer metastasis. Herein, we presented a case of a 72-year-old man, who was admitted for chest pain and with radiological evidence of lung cancer with distant metastases and a suspected localization in the right colon. On colonoscopy visual impression showed two yellowish and ulcerative lesions, one in the ascending colon and the other in the transverse colon, which were biopsied. Both were consistent on pathological report with colonic metastases. According to advanced stage of neoplasm (stage IV), patient underwent palliative chemotherapy.

Keywords: Lung cancer; Malignant neoplasm; Colonoscopy; Palliative chemotherapy; Abdominal; Neoplastic cells

Abbreviations: GI: Gastrointestinal; CT: Computed Tomography; FDG: Fluorodeoxy Glucose; SUV: Standardized Uptake Value; LC: Lung Cancer; PET-CT: Positron Emission Tomography Computed Tomography

\section{Introduction}

Gastrointestinal (GI) metastates from lung tumour are rarely reported in literature. Clinical presentation is variable and often patient with GI metastases are asymptomatic. We report a case of asymptomatic colonic metastases of lung cancer.

\section{Case Presentation}

We present a case of a 72-year-old Caucasian man who was admitted to our hospital for chest-pain and X-ray evidence of a mediastinal mass. Chest contrast enhanced computed tomography (CT) revealed a mediastinal impudence solid mass, whole sized $10 \times 11 \mathrm{~cm}$ diameter, with a disomogeneous aspect, because of the presence of a necrotic central zone, which kept contact with a segment of the left upper lung and did not invade neither aortic arch, left pulmonary trunk or chest wall; additionally, moderately enhancement of the lump, in absence of mediastinal lymphadenopathy, were also revealed. Abdominal contrast enhanced CT showed diffused and enlarged mesenterial nodes suggestive for abdominal metastasis, while contrast enhanced head CT scan showed an ipodense, contrast enhanced and disomogeneous lesion of the left frontal lobe, which was suggestive for brain metastasis.
Pathologic findings of CT guided mediastinal mass biopsy revealed a poorly differentiated adenocarcinoma of the lung. Positron emission tomography computed tomography (PET-CT) examination highlighted abnormal fluorodeoxy glucose (FDG) metabolism nodules in the mesentery, in the left adrenal gland and a nodule in the right colon wall, which have a maximum standardized uptake value (SUV) value of 12.9.

Colonoscopy revealed a focal yellowish, friable and ulcerative lesion of the proximal ascending colon with a diameter of approximate $35 \mathrm{~mm}$ (Figure 1A) and another lesion with the same visual characteristics in the proximal transverse colon, with a diameter of $20 \mathrm{~mm}$ (Figure 1B), which were biopsied both. In both samples the biopsy showed neoplastic tissue fragments consistent with a poorly differentiated tumour with solid growth pattern and small foci of necrosis (Figure 1C).

At immunohistochemistry, neoplastic cells were diffusely positive for CK7 and focally positive for TTF1, while negative for CK20 and CDX2 (Figure 1D). These features, along with clinical and radiological data, were consistent with a metastatic lesion of pulmonary origin. According to stage IV of the neoplasm, the patient's treatment plan was palliative chemotherapy. 


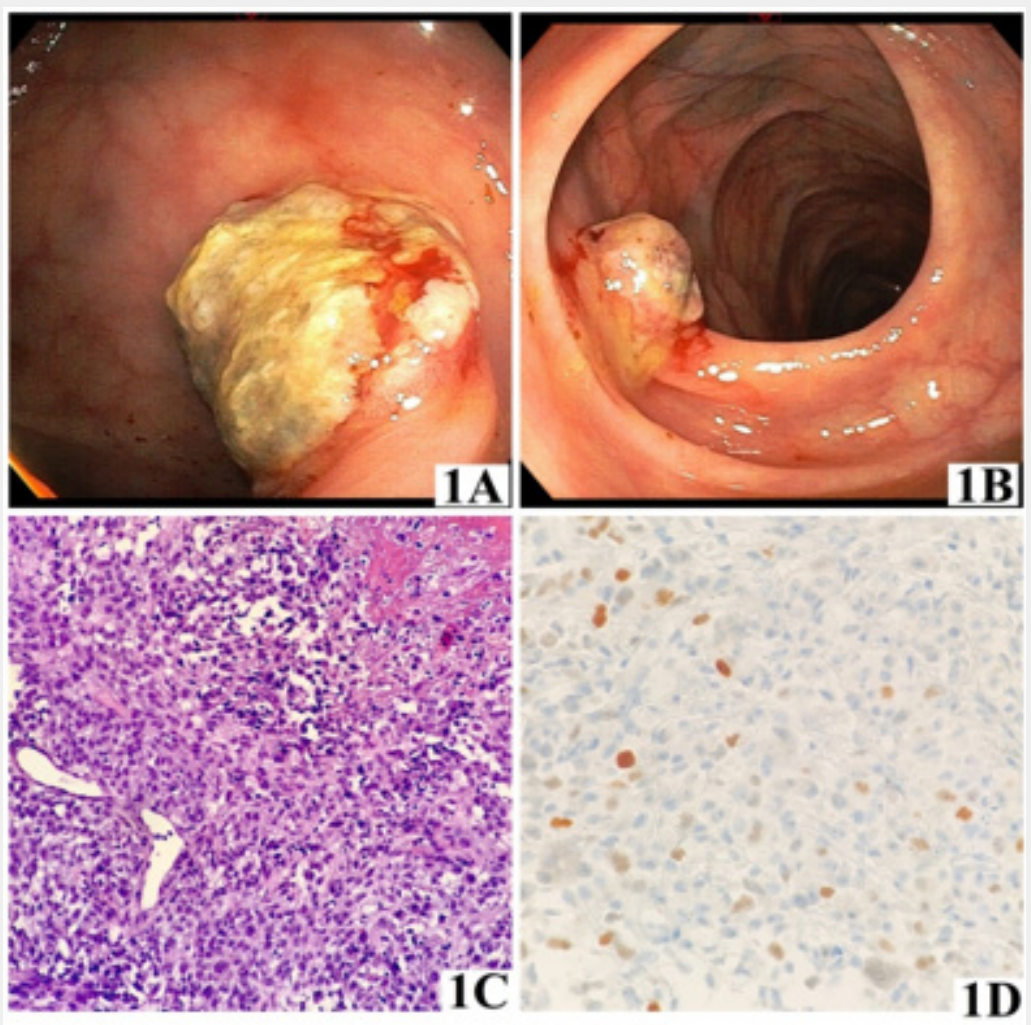

Figure 1: Colonscopy findings showing two yellowish, ulcerative and friable focal lesions, respectively in the proximal ascending colon $(1 \mathrm{~A})$ and in the proximal transverse colon (1B). Mucosal biopsy of the lesion of the ascending colon (1C) demonstrating neoplastic tissue, which appears highly cellular with foci of necrosis at the top edge; tumour cells show abundant clear cytoplasm and pleomorphic nuclei (Hematoxylin and Eosin, 40X).

Immunohistochemical staining for TTF1 of the same lesion (1D) shows scattered nuclear positivity (40X).

\section{Discussion}

Secondary metastatic lesions of the colon are a rare clinical entity. Thus are often diagnosed in patients with a known, primary neoplasm, usually deriving from the lung, breast, ovary, prostate, kidney, stomach, skin or hepatobiliary system and in most cases, may still be asymptomatic or found at autopsy [1].

Regarding lung cancer (LC), the gastrointestinal tract is an extremely rare site of metastasis and few cases have been reported in literature [2,3]. However, Antler et al. in a separate autopsy series of 423 patients, discover an overall 14\% involvement of the gastrointestinal (GI) tract from metastatic LC. Of these, a majority (46\%) were due to the infiltration of the esophageal wall, while the large and small bowel were involved in only $6 \%$ of the cases [4].

Clinical manifestations of intestinal metastasis of LC may be lower GI bleeding, anaemia, bowel obstruction, intussusception or perforation $[1,5]$. Secondary colon lesions should be suspected in patients with metastatic cancer presenting with those aforementioned lower GI symptoms [5].
On the other hand, those intestinal metastasis can be asymptomatic and detected by radiological examination, during the initial staging or follow up phase. Diagnostic tests such as CT of the abdomen can reveal thickening of the bowel [1].

Additionally, PET, which is able to detect extrathoracic metastases in approximately $25 \%$ of patients, can highlight areas of increased FDG uptake. Thus, radiological findings should have a further pathologic evalutation, especially for patients who are being considered for surgical resection or chemoradiotherapy [6].

Diagnosis of secondary disease of LC to colon can be achieved by lower GI endoscopy.

Additionally, biopsies of those lesions must be acquired to determine whether the neoplasm has metastasized from lung to colon. Indeed, although the endoscopic evidence of multiple colonic lesions in the context of metastatic malignancy is suggestive of secondary rather than primary colon cancer, immunohistochemistry is mandatory [1].

However, immunochemistry pattern for colorectal adenocarcinoma is CDX-2 positive, CK20 positive and CK7 
negative, while LC metastases pattern is topically TTF-1 positive, CK7 positive and CK20 negative [7].

\section{Conclusion}

In conclusion, LC presenting with gastrointestinal metastasis is rather rare even at advanced stages and often asymptomatic. A prompt recognition of GI symptoms or radiological signs of GI involvement and an early patient referral for endoscopic and immunohistochemistry evaluation could help to confirm or rule out intestinal metastasis of lung neoplasm in order to better define cancer stage.

\section{Acknowledgements}

We would like to show our gratitude to the Department of Pathology Service of Bellaria-Maggiore Hospital (Bologna, Italy) for the high quality of the images of histologic and immunoistochemical findings and for comments that greatly improved the manuscript.

\section{References}

1. Michail Galanopoulos, Filippos Gkeros, Christos Liatsos, Christos Pontas, Apostolis Papaefthymiou, et al. (2018) Secondary metastatic lesions to colon and rectum. Ann Gastroenterol 31(3): 282-287.
2. S Hirasaki, S Suzuki, S Umemura, H Kamei, M Okuda, et al. (2008) Asymptomatic colonic metastases from primary squamous cell carcinoma of the lung with a positive fecal occult blood test. World J Gastroenterol 14(35): 5481-5483.

3. I Gonzalez-Tallon, J Vasquez-Guerrero, MA Garcia-Mayor (2013) Colonic Metastases from Lung Carcinoma: A Case Report and Review of the Literature. Gastroenterology Res 6(1): 29-33.

4. S Antler, Y Ough, CS Pitchumoni, M Davidian, W Thelmo (1982) Gastrointestinal metastases from malignant tumors of the lung. Cancer 49(1): 170-172.

5. KP Goh, AWY Yeo, HN Koong, LLP J Ooi, WK Wong (2007) Laparotomy for acute complications of gastrointestinal metastases from lung cancer: is it a worthwhile or futile effort? Surg Today 37(5): 370-374.

6. TE Stinchcombe, MA Socinski, LM Gangarosa, AH Khandani (2006) Lung cancer presenting with a solitary colon metastasis detected on positron emission tomography scan. J Clin Oncol 24(30): 4939-4940.

7. YC Su, YC Hsu, CY Chai (2006) Role of TTF-1, CK20, and CK7 immunohistochemistry for diagnosis of primary and secondary lung adenocarcinoma. Kaohsiung J Med Sci 22(1): 14-19.

\section{Your next submission with JuniperPublishers will reach you the below assets}

- Quality Editorial service

- Swift Peer Review

- Reprints availability

- E-prints Service

- Manuscript Podcast for convenient understanding

- Global attainment for your research

- Manuscript accessibility in different formats

( Pdf, E-pub, Full Text, audio)

- Unceasing customer service

Track the below URL for one-step submission https://juniperpublishers.com/online-submission.php 